\title{
The effect of a D2-specific dopamine agonist (Pramipexole) on response time in early Parkinson's disease patients
}

\author{
DANIEL B. WILLINGHAM, JAMES P. BENNETT, \\ NEVIN J. GREENBERG, and ELKE ROST-RUFFNER \\ University of Virginia, Charlottesville, Virginia
}

\begin{abstract}
The long-term use of dopamine replacement therapy leads to dyskinesias in some Parkinson's disease (PD) patients, which may be specific to the D1 subtype of dopamine (DA) receptor. The purpose of this study was to test the effectiveness of a D2-specific DA agonist, Pramipexole, in PD patients. In a double-blind design, 6 patients took increasing doses of Pramipexole over the course of 24 weeks, and were tested in a total of 15 sessions on four measures of movement efficacy: tapping speed, simple response time, choice response time, and sequencing. Compared with 3 placebo control patients, those on active medication showed faster tapping speeds and simple response times. Choice response time and sequencing were not affected by medication status. The D2 subtype of DA receptor may be especially important in movement speed, and may contribute less to more cognitive aspects of movement.
\end{abstract}

The hallmark of Parkinson's disease (PD) is the death of cells in the substantia nigra, pars compacta, and a resultant loss of dopamine (DA) to the striatum. The behavioral manifestations of the disease are tremor, rigidity, and bradykinesia, as well as cognitive effects, which may be subtle early in the disease, but which can progress to global dementia (Growdon \& Corkin, 1986).

Dopamine replacement therapy is effective in relieving some of the motor symptoms of PD, especially bradykinesia. In particular, PD patients treated with levodopa ("on") show a significant improvement in movement time compared with that when they are not treated with levodopa ("off"), but there is less improvement in movementinitiation time (Velasco \& Velasco, 1973). A number of researchers have investigated the effect of levodopa on motor planning by comparing simple and choice response times. A simple response-time task presumably allows for motor planning, because the response is identical on each trial, whereas a choice response-time task does not allow for motor planning, because the appropriate response is not known until the stimulus appears. Levodopa does not influence simple response time (Jahanshahi, Brown, \& Marsden, 1992; Pullman, Watts, Juncos, Chase, \& Sanes, 1988; Starkstein, Esteguy, Berthier, Garcia, \& Leiguarda,

This research was supported by a grant from UpJohn Pharmaceutical. The authors thank Susan Dietrich and the staff at the Clinical Research Center at the University of Virginia Hospital for their assistance, the patients who so willingly participated in this research, and two anonymous reviewers who provided helpful comments on an earlier version of this article. Correspondence concerning this article should be addressed to D. B. Willingham, Department of Psychology, University of Virginia, 102 Gilmer Hall, Charlottesville, VA 22903 (e-mail: willingham@ virginia.edu).
1989; Velasco \& Velasco, 1973) but does influence choice response time (Brown et al., 1993; Pullman et al., 1988; but see Jahanshahi et al., 1992). This result may be interpreted to mean that levodopa does not aid in motor planning.

Although levodopa is an effective treatment for PD, there is a drawback to its long-term use. After several years, many patients find a loss of efficacy toward the end of the dosing interval and later develop dyskinesias toward the peak of levodopa action (Mouradian et al., 1988). These dyskinesias are involuntary hypokinetic choreoathetotic, dystonic, or myoclonic movements. This problem may progress such that the patient shows dyskinesia during much of the "on" phase and then rapid onset of bradykinesia as the dose wears off. There are several possible reasons for these changes in response, including sensitization of DA receptors (Engber, Susel, Juncos, \& Chase, 1988), a change in basal ganglia output neurons themselves (Gerfen et al., 1990), or progressively rapid fluctuations in synaptic levels of DA in patients with advanced PD (Orosz \& Bennett, 1992).

The onset and progressive worsening of dyskinesias may not be a necessary consequence of the treatment of symptoms in PD, however, because the two effects may be separable. Clozapine selectively disrupts the extent to which levodopa produces dyskinesias (Bennett, Landow, $\&$ Schuh, 1993). This effect may be due to the fact that Clozapine has a high affinity for the D2 subtype of DA receptor and low affinity for the D1 subtype (Murray \& Waddington, 1990).

The hope, then, is that a D2-specific agonist would offer substantial symptom relief without the development of dyskinesias. Work with animal models of PD indicate that relief of symptoms is available from agonists that selectively occupy the D2 subtype of DA receptor. Motor be- 
Table 1

Parkinson's Disease Patient-Subject Characteristics

\begin{tabular}{|c|c|c|c|c|c|}
\hline Patient & Condition & Age & $\begin{array}{l}\text { Education } \\
\text { (Years) }\end{array}$ & $\begin{array}{c}\text { Stage } \\
\text { (Hoehn \& Yahr, 1967) }\end{array}$ & Years PD \\
\hline 1 & Active & 70 & 16 & 1.5 & 5 \\
\hline 2 & Active & 57 & 12 & 2 & 5 \\
\hline 3 & Active & 67 & 12 & $i$ & 4 \\
\hline 4 & Active & 61 & 16 & 1.5 & 4 \\
\hline 5 & Active & 73 & 16 & 1.5 & 4 \\
\hline 6 & Active & 74 & 18 & 1 & 5 \\
\hline 7 & Placebo & 68 & 15 & 2 & 4 \\
\hline 8 & Placebo & 74 & 12 & 1 & 6 \\
\hline 9 & Placebo & 50 & 16 & 1 & 5 \\
\hline
\end{tabular}

havior can be stimulated in DA-depleted rats and primates through selective D2-specific DA agonists, although still more effective treatment stimulates D1 and D2 subtypes simultaneously (Calon et al., 1995; Carlson, Bergstrom, \& Walter, 1987; Rouillard, Bedard, \& Dipaolo, 1990; Walters, Bergstrom, Carlson, Chase, \& Braun, 1987).

Comparable data on humans are limited. In one study, Hubble et al. (1995) investigated the efficacy of Pramipexole, a highly specific D2 agonist, in humans. They reported no effect of Pramipexole compared with placebo control subjects on the motor examination part of the Unified Parkinson's Disease Rating Scale (UPDRS). They did report significant improvement on the Activities of Daily Living part of the UPDRS. Hubble et al. limited data collection to scores on the UPDRS.

The purpose of the work reported here was to evaluate the efficacy of Pramipexole through the use of formal measures of movement. The behavioral measures were a measure of motor speed (tapping), a measure of simple planning (simple response time vs. choice response time), and a measure of motor sequencing.

\section{METHOD}

\section{Overview}

The work reported here was part of a larger study on the effects of oral Pramipexole in PD. Each patient's participation in the larger study lasted approximately 13 months.

The study used a placebo control and was double blind. The subjects were randomly assigned to take either active medication or placebo. Those on active medication took equal t.i.d. doses of Pramipexole, starting at $0.375 \mathrm{mg}$ and increasing weekly by $0.75 \mathrm{mg}$ to a maximum (after 7 weeks) of $4.5 \mathrm{mg}$. The subjects were encouraged to increase to the highest dose, but were allowed to remain on a lower dose if they (or the physician) noted side effects. They stayed on this maximum dose for 6 months.

In the work reported here, the subjects were tested at weekly intervals for 8 weeks, biweekly for 12 weeks, and then in a final session 4 weeks later. Thus, there was a total of 15 sessions over the course of $4 \frac{1}{2}$ months. Testing sessions were always conducted in the morning after the subjects had taken their morning doses. At each session, the subjects were evaluated by a physician, had their blood drawn, and performed the four tasks described below.

\section{Subjects}

A total of 12 subjects participated in this study. They were required to have had no prior levodopa treatment totaling 180 days or more and none within 60 days of the initial evaluation. Subjects were excluded from the study if they had atypical PD (i.e., due to drugs or metabolic disorder), or if they had seizure disorder, recent surgery, or a history of major depression. The disease severity of all patients was rated between Stage I and Stage II on the Hoehn and Yahr (1967) scale at the start of the study, and all of the patients were capable of easily understanding the instructions for the study.

Twelve subjects initially participated, but 3 of them dropped out before the study was complete. All 3 reported that they were not getting symptom relief from the medication and that it would be too difficult to continue. When the drug-labeling code was later broken, it was determined that all 3 had been receiving the placebo. Subject characteristics are shown in Table 1 . The placebo and active medication groups did not differ in terms of age, education, disease severity, or disease duration (all $t s \leq 1.0$ ).

\section{Procedure}

Tapping. In the tapping task, the subjects were asked to tap a response key (Radio Shack No. 275-1566) on a response board measuring $34.5 \times 23.5 \mathrm{~cm}$. They were instructed to tap using the index finger of the dominant hand as quickly as possible. There was no cue to begin tapping; the task was self-initiated. The subjects performed 25 responses, whereupon a tone sounded and the words "Rest Break" appeared on the screen for $60 \mathrm{sec}$. The subjects completed three sets of 25 trials at each testing session. No feedback was provided. Response time (RT) was recorded to the nearest $\pm 1.4 \mathrm{msec}$. The dependent measure of interest was the intertap interval, that is, the time between successive taps.

Simple response time (SRT). In the SRT task, subjects saw a square $(3 \times 3 \mathrm{~cm})$ at the center of a Macintosh computer screen $(33 \mathrm{~cm}$ diagonal). Then they saw a warning signal (the word "Ready") followed by a pause of random duration $(500-1,500 \mathrm{msec})$, after which the signal to respond (GO signal) appeared, and the empty square became filled in black. The subjects responded by using the index finger of the preferred hand. RT was recorded from the time the GO signal appeared until the key was depressed.

Choice response time (CRT). In the CRT task, subjects saw four squares $(3 \times 3 \mathrm{~cm})$ on the computer monitor, centered vertically, and equidistant from one another and from the screen edges horizontally. At a viewing distance of $65 \mathrm{~cm}$, the distance between adjacent stimuli was approximately $4^{\circ}$ of visual angle and the display covered just under $12^{\circ}$ of visual angle. Trials were conducted as in the SRT task in that the subjects saw a warning signal followed by a pause of $500-1,500 \mathrm{msec}$, followed by a GO signal. The GO signal was a darkening of one of the four squares, and a subject was required to press the key directly below the darkened square. The subject used the index and middle fingers of each hand to respond. Center-to-center distance of the keys was $4 \mathrm{~cm}$. The subjects heard a brief $(120-\mathrm{msec})$ tone if they responded with the wrong key, but there was no feedback regarding RTs. There were 25 trials at each of the four locations, appearing in random order, for a total of 100 trials at each testing session. There was a 1 -min rest break after 50 trials.

Sequencing. In the sequencing task, the stimuli and responses were the same as those used in the CRT task. In this task, the subjects first watched a sequence of one, two, or three positions, indicated by a sequence of the squares becoming darkened. Each square was darkened for $500 \mathrm{msec}$, and the interstimulus interval was $250 \mathrm{msec}$. At the end of the sequence, the message "Ready" appeared for a random period between 1,000 and 3,000 msec, followed by the word "Go," whereupon the subjects were to produce the sequence on the response keys as quickly as possible. As they pushed the keys, each response was echoed on the monitor by the corresponding square's becoming filled in black.

\section{RESULTS}

Outliers were removed from the RTs by eliminating the five fastest and five slowest RTs from each task for each testing session. A mean was calculated from the remain- 


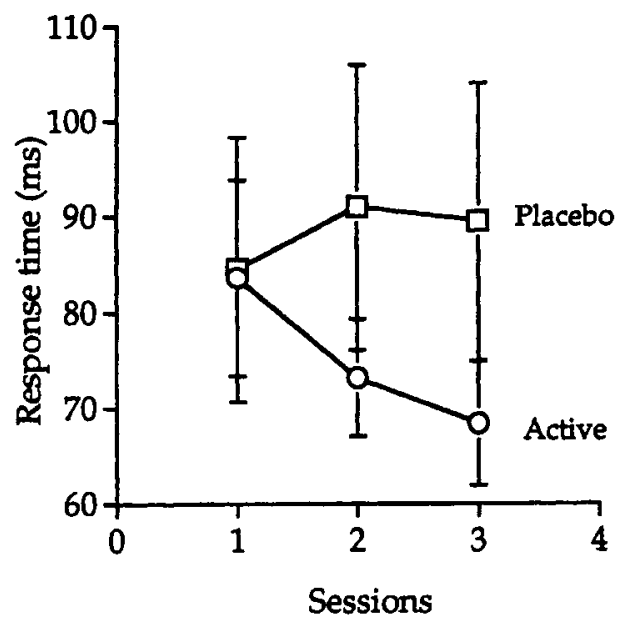

Figure 1. Mean response times across sessions, separated by condition, for the tapping task. Error bars are standard errors of means.

ing RTs as a summary statistic for each session. To reduce the variability of the data, these 15 means were further reduced by taking the mean of every five successive sessions to yield three scores that summarized performance across time.

\section{Tapping}

The results of the tapping task appear in Figure 1. There was no main effect of condition (active vs. placebo) or of session $(F \mathrm{~s}<1.0, p \mathrm{~s}>.20)$, but the interaction was marginally reliable $[F(2,14)=3.7, p=.052]$. This marginal interaction is clearly due to an improvement in RT across sessions for the active group, and the lack of a corresponding improvement for the placebo group.

\section{Simple Response Time}

The results of the SRT task appear in Figure 2. There was no effect of condition $(F<1.0, p>.20)$. There was an effect of session $[F(2,14)=6.2, p<.05]$, but this effect must be viewed in the context of the interaction effect, which was also reliable $[F(2,14)=5.9, p<.05]$. Inspection of Figure 2 makes it clear that the main effect of session is due entirely to the increasing RTs of the placebo group across sessions; the active medication group does not respond more slowly across sessions. The progressive slowing of the placebo group across sessions may mean that the SRT task is especially susceptible to a placebo effect, which diminishes with time.

\section{Choice Response Time}

The results of the CRT task appear in Figure 3. There was no effect of condition $(F<1.0, p>.20)$ or of session $[F(2,14)=2.1, p=.16]$. The interaction of condition and session appears similar to that seen in the SRT task, but this effect did not approach reliability $[F(2,14)=2.4$, $p=.13]$. Thus, unlike in the SRT task, treatment with
Pramipexole appears not to affect CRT. Subjects made very few errors on this task (fewer than $3 \%$ ) and the medication condition had no effect on the number of errors.

\section{Sequencing}

On similar sequencing tasks, normal subjects respond more slowly on the initial keypress than on successive positions of the sequence. Figure 4 shows RTs for sequences of lengths 1, 2, and 3 positions for the placebo and control conditions. It is clear that the patients on active medication show a very robust effect of sequence position, with the first keypress being slower than successive presses. Patients in the placebo group show the same pattern, but with smaller mean differences and more variability. There was a reliable main effect of sequence position on $\operatorname{RT}[F(5,35)=$ $3.1, p<.05$ ], but there was not a statistical difference in the responses of placebo and active medication subjects across the different sequences $[F(5,35)=.68, p>.20]$.

There was a reliable interaction of condition and session, however $[F(10,70)=2.8, p<.01]$, which is shown in Figure 5. Placebo subjects improved RTs with practice, whereas the active medication subjects did not. This effect was consistent across the different sequences $(F<1.0)$.

Subjects made few errors (accuracy was $93 \%$ or greater for all responses), but there was a consistent effect of sequence type $[F(5,35)=13.9, p<.001]$, whereby the responses of length 1 or length 2 were very accurate, as were the initial responses for the sequences of length 3 (all 96\%-99\% correct). The second and third responses for sequences of length 3 were less accurate (93\% correct for each). There was also a reliable interaction of this effect with sessions $[F(10,70)=2.3, p<.05]$. Subjects' accuracy on the second and third responses for sequences of length 3 improved across sessions from about $91 \%$ to about $95 \%$, whereas accuracy for other responses did not change, possibly because accuracy was so high from the start.

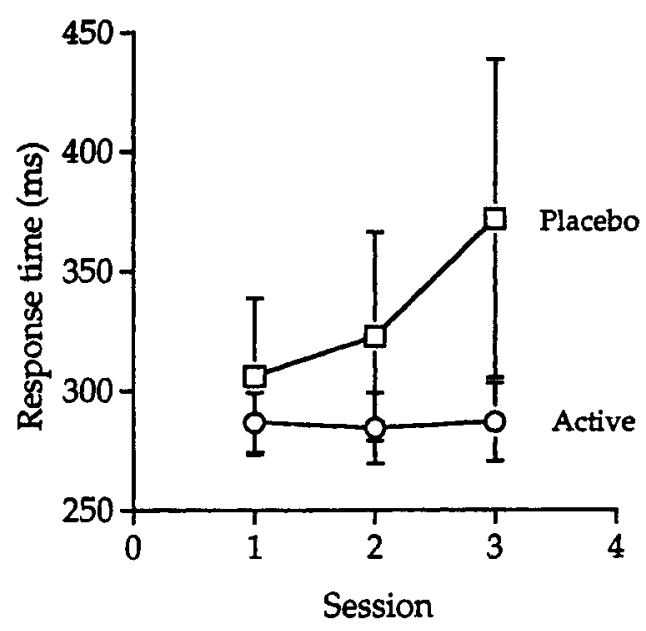

Figure 2. Mean response times across sessions, separated by condition, for the simple response-time task. Error bars are standard errors of means. 


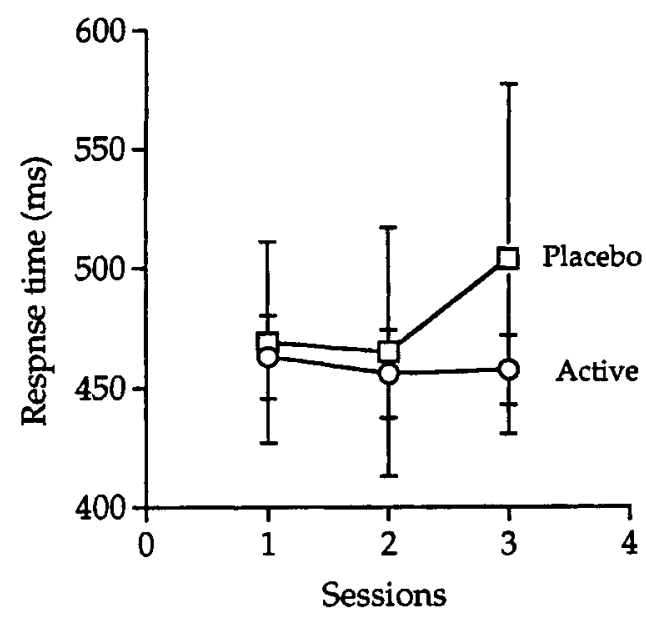

Figure 3. Mean response times across sessions, separated by condition, for the choice response-time task. Error bars are standard errors of means.

\section{DISCUSSION}

Pramipexole affected the performance of two tasks: tapping and the simple RT task. There was not a reliable effect on the choice RT task or on the sequencing task, although these negative results must be viewed in the context of the limited power afforded by the study.

The present results may be compared to those of Hubble et al. (1995), who reported only a trend $(p=0.1)$ toward improvement for patients taking Pramipexole on the motor scale of the UPDRS. Their patients were comparable to those tested here in terms of disease severity. The dosages were also comparable, as was the length of the study. A key difference may be the manner in which motor signs were evaluated. The UPDRS motor scale provides an overall measure of motor performance that includes measures at rest (e.g., tremor, rigidity) as well as ability to execute different types of movements (e.g., gait movements, rapid alternating movements, simple tapping movements). Our data indicate that Pramipexole may not be equally effective in relieving all movement-related Parkinsonian symptoms. Thus, Hubble et al. may have observed only a trend in improvement in the UPDRS motor scale because the effect of the medication on some measures was lost when combined with the other measures that Pramipexole does not affect.

It is noteworthy that the medication effect was not uniform across the different tasks. The effect was reliable (or marginally so) in the tapping and SRT tasks, but not in the CRT or sequencing tasks. It may be relevant that the tapping and SRT tasks do not require response selection on the part of the subject. The premium in these tasks is on simple motor speed. In the CRT task and the sequencing tasks, much of the total time the subject uses to respond is not devoted to generating the motor response, but rather to the more cognitive process of selecting how to respond. In the tapping and the SRT tasks, the subject makes the same movement on each trial, and RTs are quite short, as shown in Figures 1 and 2. In the CRT and sequencing tasks, a different response must be programmed on each trial, and RTs are quite a bit longer. Thus, it may be that Pramipexole speeds motor time but does not affect the more cognitive process of selecting the appropriate movement to respond to a given stimulus. The effect of Pramipexole is therefore easier to detect in tasks that are almost exclusively movement tasks, such as the SRT and tapping tasks. Under this interpretation, Pramipexole does affect the sequencing and CRT tasks, but this effect is difficult to detect, because the RT to these tasks is so long and so variable, due to the cognitive requirements of the task. It is also possible that Pramipexole has a smaller impact on these tasks, and that there was not sufficient power in this study to detect it.

The results reported here-that SRT is aided but CRT is not - contrast with results reported for the effect of levodopa on these tasks. A number of studies report the opposite effect: levodopa does not affect SRT (Jahanshahi et al., 1992; Pullman et al., 1988; Starkstein et al., 1989; Velasco \& Velasco, 1973) but does improve CRT (Brown et al., 1993; Pullman et al., 1988; but see Jahanshahi et al., 1992). There are several possible interpretations of this difference. First, it should again be emphasized that power in this experiment was limited by the small number of subjects, and so it is possible that there would have been an effect of Pramipexole on the CRT and sequencing tasks had there been more patients. Still, the limited power makes the observation of the effect on SRT all the more remarkable.

It is possible that the difference between previous studies and the present work lies in the different action of levodopa and Pramipexole. Levodopa is not selective for the two major subtypes of DA brain receptors (D1 or D2), whereas Pramipexole is highly selective for the D2 sub-

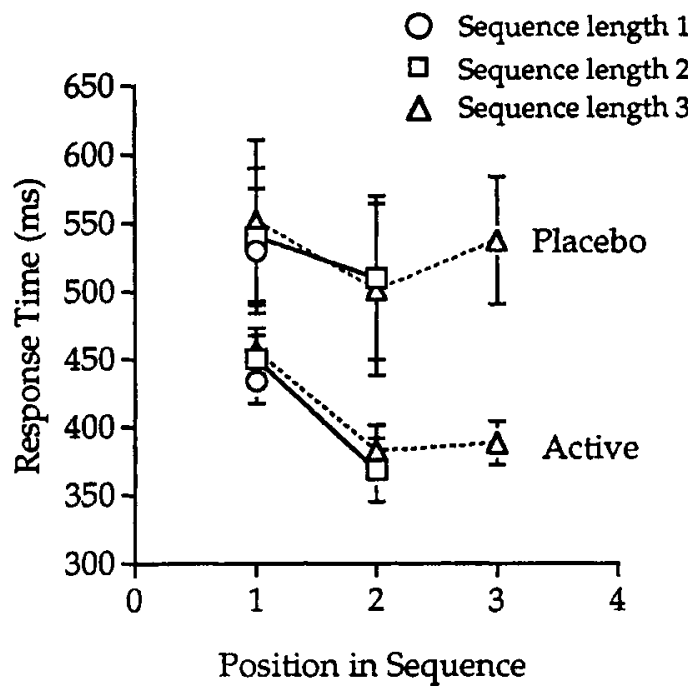

Figure 4. Mean response times, across sequence positions, separated by condition, for the sequencing task. Error bars are standard errors of means. 


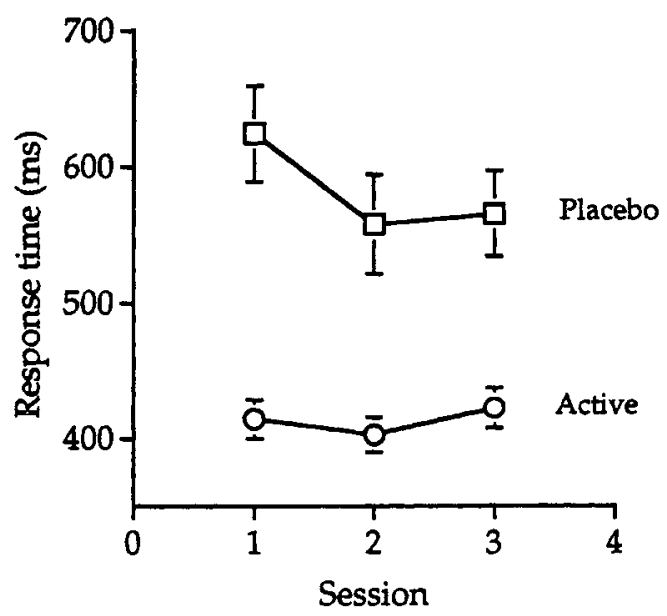

Figure 5. Mean response times across sessions, separated by condition, for the sequencing task. Error bars are standard errors of means.

type. D1 receptors, which Pramipexole would not influence, are prominent in the frontal cortex, but the concentration of D2 receptors is relatively low (Goldman-Rakic, Lidow, \& Gallager, 1990). Frontal cortex is also known to be important in the high-level selection of motor behaviors (e.g., Barone \& Joseph, 1989; Mann, Thau, \& Schiller, 1988). One might therefore expect that levodopa would benefit tasks that demanded that the patient select from among several possible motor behaviors (e.g., tasks like the CRT task and the sequencing task), but that Pramipexole would not, and indeed, that is the pattern that we observed. On the other hand, this account does not readily explain why levodopa does not speed SRT whereas Pramipexole does.

A possible explanation for the failure to observe an effect of levodopa on SRT in previous studies is that it may be due to the methodology of the testing. Previous studies examining SRT and CRT tasks have used within-subjects designs, and therefore did not include a placebo control group (Jahanshahi et al., 1992; Starkstein et al., 1989; Velasco \& Velasco, 1973). Such designs confound possible drug effects and placebo effects, because patients are well aware of whether they are on or off medication. In the present study, we observed a significant placebo effect in the SRT task, as shown in Figure 2. Patients taking placebo respond quite quickly in early sessions, but the placebo effect diminishes across sessions and their RTs increase. Notice that the patients taking active medication do not improve their RTs across sessions, despite the fact that their dosage is increasing across sessions. A ready explanation for this is that although they, too, show fast RTs due to a placebo effect in the early sessions when the dosage is quite low, as the placebo dissipates across weeks, the increasing dosage of Pramipexole allows the active-medication subjects to maintain their fast RTs. The tapping task may not be as susceptible to a placebo effect, and so Pramipexole speeds responses in this task as dosage increases.
In summary, the failure to observe an effect of Pramipexole on CRT may be due to the fact that it is selective to D2 dopamine receptors, and the prefrontal region subserving motor-response selection is rich in D1 receptors. As for the SRT task, other studies examining levodopa may have failed to show a drug effect because it was masked by a placebo effect; had previous studies tested subjects across multiple sessions so that the placebo effect dissipated, a drug effect would have been observed, as it was in this study.

The primary purpose of this study was to investigate the efficacy of a selective D2 dopamine agonist in relieving symptoms of Parkinsonism in humans. The data reported here indicate that such an agent is effective in alleviating bradykinesia, but may not influence more cognitive aspects of movement planning.

\section{REFERENCES}

Barone, P., \& Joseph, J. P. (1989). Role of the dorsolateral prefrontal cortex in organizing visually guided behavior. Brain, Behavior \& Evolution, 33, 132-135.

Bennett, J. P., JR., Landow, E. R., \& Schuh, L. A. (1993). Suppression of dyskinesias in advanced Parkinson's disease: II. Increasing daily clozapine doses suppress dyskinesias and improve Parkinsonism symptoms. Neurology, 43, 1551-1555.

Brown, V. J., Schwartz, U., Bowman, E. M., Fuhr, P., Robinson, D. L., \& HalleTt, M. (1993). Dopamine dependent reaction time deficits in patients with Parkinson's disease are task specific. Neuropsychologia, 31, 459-469.

Calon, F., Goulet, M., Blanchet, P. J., Martel, J. C., Peircey, M. F., BEDARD, P. J., \& DiPAOLO, T. (1995). Levodopa or D2 agonist induced dyskinesia in MPTP monkeys: Correlation with changes in dopamine and GABA receptors in the striatopallidal complex. Brain Research, 680, 43-52.

Carlson, J. H., Bergstrom, D. A., \& Walter, J. R. (1987). Stimulation of both D1 and D2 receptors appears necessary for full expression of postsynaptic effects of dopamine agonists: A neurophysiological study. Brain Research, 400, 205-218.

Engber, T. M., Susel, Z., Juncos, J. L., \& Chase, T. N. (1988). Continuous and intermittent levodopa differentially affect rotation induced by D-1 and D-2 dopamine agonists. European Journal of Pharmacology, 168, 291-298.

Gerfen, C. R., Engber, T. M., Mahan, L. C., Susel, Z., Chase, T. N., Monsma, F. J., \& SibLEY, D. R. (1990). D1 and D2 dopamine receptorregulated gene expression of striatonigral and striatopallidal neurons. Science, 250, 1429-1432.

Goldman-Rakic, P. S., Lidow, M. S., \& Gallager, D. W. (1990). Overlap of dopaminergic, adrenergic, and serotoninergic receptors and complementarity of their subtypes in primate prefrontal cortex. Journal of Neuroscience, 10, 2125-2138.

Growdon, J. H., \& CorKIN, S. (1986). Cognitive impairments in Parkinson's disease. In M. D. Yahr \& K. J. Bergman (Eds.), Advances in neurology (Vol. 45, pp. 382-393). New York: Raven Press.

HoEHN, M. M., \& YAHR, M. D. (1967). Parkinsonism: Onset, progression and mortality. Neurology, 17, 427-442.

Hubble, J. P., Koller, W. C., Cutler, N. R., Sramek, J. J., Friedman, J., Goetz, C., Ranhosky, A., KorTs, D., \& Elvin, A. (1995). Pramipexole in patients with early Parkinson's disease. Clinical Neuropharmacology, 18, 338-347.

Jahanshahi, M., Brown, R. G., \& Marșen, C. D. (1992). The effect of withdrawal of dopaminergic medication on simple and choice reaction time and the use of advance information in Parkinson's disease. Journal of Neurology, Neurosurgery \& Psychiatry, 55, 1168-1176.

Mann, S. E., Thau, R., \& Schiller, P. H. (1988). Conditional taskrelated responses in monkey dorsomedial frontal cortex. Experimental Brain Research, 69, 460-468. 
Mouradian, M. M., Juncos, J. L., Gabbrini, G., Schlegel, J., BARTKO, J. J., \& ChASE, T. N. (1988). Motor fluctuations in Parkinson's disease: Central pathophysiological mechanisms, Part II. Annals of Neurology, 24, 372-378.

MurRay, A. M., \& Waddington, J. L. (1990). The interaction of clozapine with dopamine D1 versus dopamine D2 receptor-mediated function: Behavioral indices. European Journal of Pharmacology, 186, 79-86.

Orosz, D., \& BENNETT, J. P., JR. (1992). Simultaneous microdialysis in striatum and substantia nigra suggests that the nigra is a major site of action of l-dihydroxyphenylalanine in the "hemiparkinsonian" rat. Experimental Neurology, 115, 388-393.

Pullman, S. L., WatTs, R. L., Juncos, J. L., Chase, T. N., \& Sanes, J. N. (1988). Dopaminergic effects on simple and choice reaction time performance in Parkinson's disease. Neurology, 38, 249-254.

Rouillard, C., Bedard, P. J., \& DipaOlo, T. (1990). Behavioral and biochemical effect of chronic treatment of MPTP-monkeys with bromocriptine alone or in combination with SKF 38393. European Journal of Pharmacology, 185, 209-215.

Starkstein, S. E., Esteguy, M., Berthier, M. L., Garcia, H., \& LeiGUARDA, R. (1989). Evoked potentials, reaction time, and cognitive performance in on and off phases of Parkinson's disease. Journal of Neurology, Neurosurgery \& Psychiatry, 52, 338-340.

Velasco, F., \& VelasCo, M. (1973). A quantitative evaluation of the effects of L-DOPA on Parkinson's disease. Neuropharmacology, 12, 89-99.

Walters, J. R., Bergstrom, D. A., Carlson, J. H., Chase, T. N., \& BraUN, A. R. (1987). D1 receptor activation required for postsynaptic expression of D2 agonist effects. Science, 236, 719-722.

(Manuscript received May 9, 1997;

revision accepted for publication August 18,1997 .) 